\title{
Massive bilateral adrenal mass with adrenal insufficiency: a case report of primary adrenal lymphoma
}

\author{
Seher Çetinkaya Altuntaş๑, Ŭ̆ur Avcı® \\ Department of Internal Medicine, Division of Endocrinology, Recep Tayyip Erdoğan University Faculty of Medicine, Rize, Turkey
}

\begin{abstract}
Primary Adrenal Lymphoma (PAL) is extremely rare and usually occurs in men in the $6^{\text {th }}-7^{\text {th }}$ decade as bilateral, diffuse large B-cell lymphoma (DLBCL). Here, an 80-year-old patient admitted to the urology outpatient clinic with flank pain and bilateral adrenal mass detected on ultrasound is presented. Positron Emission TomographyComputed Tomography (PET-CT) was planned for the patient who was referred to the endocrine outpatient clinic. The PET-CT scan revealed lobulated-contoured masses containing necrotic areas with a size of $7.4 \times 5.5 \times 9.8 \mathrm{~cm}, 19$ Hounsfield Unit (HU), and SUVmax value of 23.9 the right adrenal, and with the size of $8.4 \times 8.7 \times 10.8 \mathrm{~cm}, 28 \mathrm{HU}, \mathrm{SUVmax}$ value of 27.3 in the left adrenal. These masses were reported to be not compatible with metastasis and suggested a tumor of the adrenal origin or bilateral adrenocortical carcinoma. In laboratory tests, since Adrenocorticotropic hormone level 291-592 pg/mL (high) and Cortisol level was 7.5$9.5 \mathrm{mcg} / \mathrm{dL}$ (low), bilateral adrenalectomy was performed considering adrenocortical cancer primarily. Diffuse large B cell lymphoma was determined as the result of the pathology. Postoperative hydrocortisone and fludrocortisone treatment was initiated for the patient immediately. The patient was transferred to the hematology inpatient clinic to receive Rituximab, Cyclophosphamide, Doxorubicin, Vincristine, and Prednisolone chemotherapy. In this case report, we aimed to emphasize that the differential diagnosis should be made well in massive bilateral adrenal masses. Although the diagnosis is challenging, clinicians should be alert in diagnosing lymphoma in bilateral, massive adrenal masses with adrenal insufficiency. Since this disease's prognosis is poor and aggressive, a histopathological diagnosis should be obtained, and treatment should be initiated as soon as possible.
\end{abstract}

Keywords: Adrenal insufficiency, non-Hodgkin's lymphoma, primary adrenal lymphoma

A 1though lymphomas generally consist of lymphoid tissue, less than $25 \%$ of cases are of extranodal origin. Primary Adrenal lymphoma (PAL) is extremely rarely seen as less than $1 \%$ of all cases. It is usually seen as bilateral and non-germinal centered diffuse large B-cell lymphoma (DLBCL) in males in the 6 th and 7 th decade [1]. Although the etiology remains unknown, the Epstein-Barr virus, genetic defects in P53 and C-kit, and immune system dysfunction are considered responsible [2].
Here, an 80-year-old patient admitted with a flank pain complaint was operated considering adrenal carcinoma in the pre-diagnosis and diagnosed with bilateral high-grade B-cell lymphoma as the result of pathology is presented.

\section{CASE PRESENTATION}

An 80-year-old female patient with no known co-

2. How to cite this article: Çetinkaya Altuntaş $S$, Avcı U. Massive bilateral adrenal mass with adrenal insufficiency: a case report of primary adrenal lymphoma. Eur Res J 2022;8(1): 126-130. DOI: 10.18621/eurj.821870

Address for correspondence: Seher Çetinkaya Altuntaş, MD., Recep Tayyip Erdoğan University Faculty of Medicine, Department of Internal Medicine, Division of Endocrinology, 53100,Rize, Turkey.E-mail: drsehercetinkaya@hotmail.com, Tel: +90 505 7049748, Faks:+90 34642130491 
morbidities applied with left flank pain, fatigue, and weakness to the urology outpatient clinic. The ultrasonography examination revealed heterogeneous hypoechoic lesions with the size of $81 \times 37 \mathrm{~mm}$ in the right adrenal and $85 \times 50 \mathrm{~mm}$ in the left adrenal, and the patient referred to the endocrine outpatient clinic. In the patient's physical examination evaluated in our clinic, her blood pressure was $90 / 60 \mathrm{mmHg}$, and her pulse was $95 / \mathrm{min}$. The examinations of all systems were normal, and there was no organomegaly. No abnormalities were found in routine biochemistry and complete blood count tests. The hormonal test results of the patient are presented in Table 1. With a pre-diagnosis of adrenal insufficiency, a $250 \mathrm{mcg}$ intravenous synacthen stimulation test was performed on the patient. The adrenal insufficiency diagnosis was made based on the cortisol levels (Table 2). The Magnetic Resonance Imaging (MRI) of the patient was reported as space-occupying lesions with a size of approximately $70 \times 50 \mathrm{~mm}$ in the left adrenal region, approximately $58 \times 36 \mathrm{~mm}$ in the right adrenal region, slightly hypointense in T1, slightly hyperintense in T2, and a slight signal loss in out phase sequences (Figs. 1 and 2). Positron Emission Tomography-Computed Tomography (PET-CT) was requested to examine malignancy due to the mass's size, the patient's age, and the clinical presentation. PET-CT resulted as masses in right adrenal with $7.4 \times 5.5 \times 9.8 \mathrm{~cm}$ size, 19 Hounsfield Unit (HU), SUV max: 23.9, and left adrenal lobulated contoured $8.4 \times 8.7 \times 10.8 \mathrm{~cm}$ in size, 28 HU, SUVmax: 27.3. Necrotic areas were noticeable in both masses. Bilateral adrenal masses were not compatible with metastasis and were reported as either tumors of the adrenal origin or bilateral adrenocortical carcinoma. An endocrine department consultation was requested in the preoperative period. Considering the bilateral adrenal carcinoma and primary adrenal insufficiency first, the patient was operated under steroid infusion and bilateral adrenalectomy was performed. No complications occurred during or after the operation. Postoperative hydrocortisone $15 \mathrm{mg} /$ day and fludrocortisone $0.1 \mathrm{mg} /$ day treatment was started due to primary adrenal insufficiency. The patient's pathology result was reported as "Neoplastic cells are antigenically positive with CD20, CD79a, mum1, bc16, and PAX5, negative with CD3, CD5, CD10, Tdt, CyclinD1, MPO, and CD34. Ki-67 proliferation index is around $90 \%$, and no loss with BCL-2. Morphological and immunohistochemical findings are consistent with diffuse large B cell lymphoma infiltration, germinal off-center". The patient was transferred to the hematology inpatient clinic. R-CHOP (Rituximab - Cyclophosphamide + Doxorubicin + Vincristine + Prednisolone) chemotherapy was initiated.

\section{Table 1. Preoperative hormonal test results}

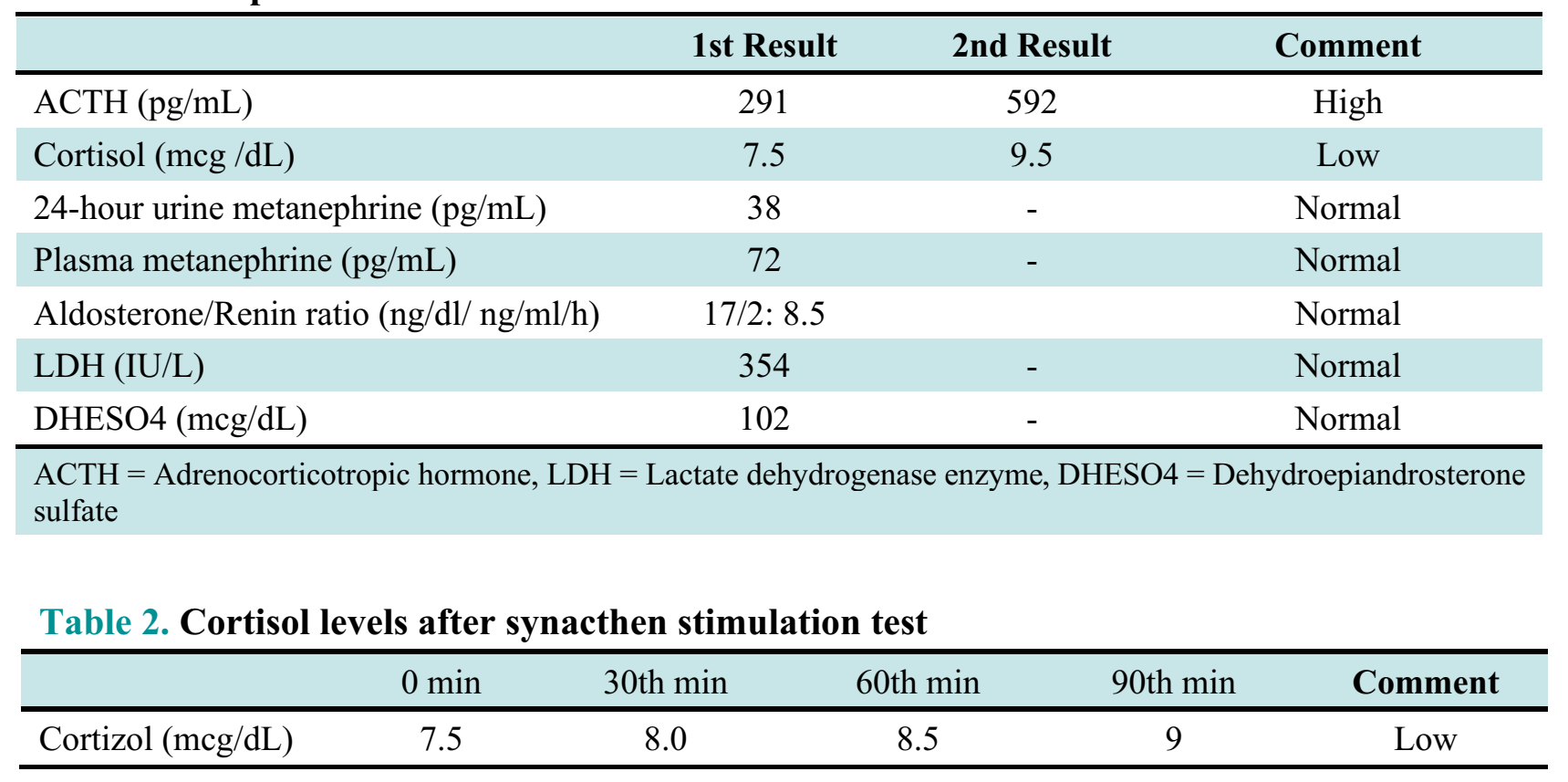




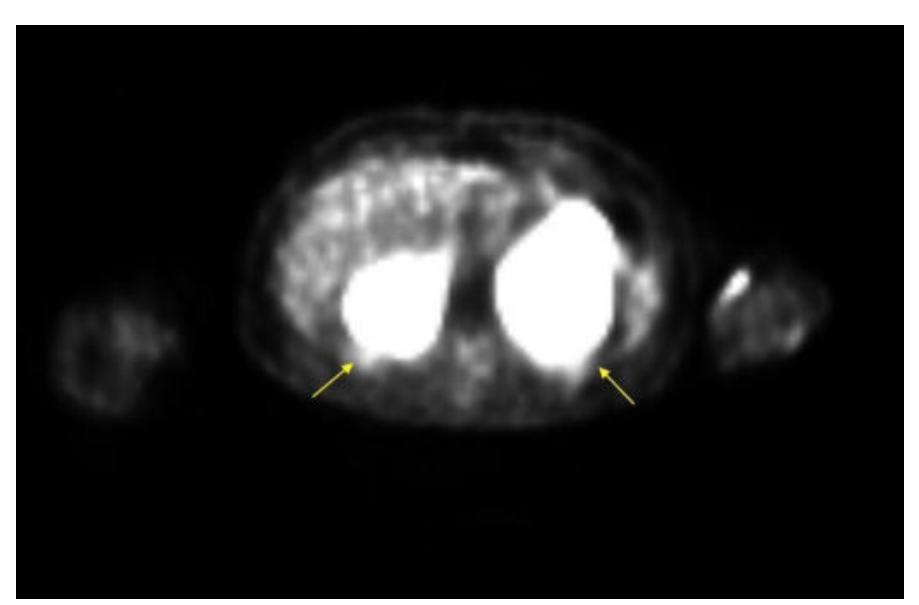

Fig.1. In diffusion-weighted images, mass lesions showing diffusion restriction are observed in the bilateral adrenal glands.

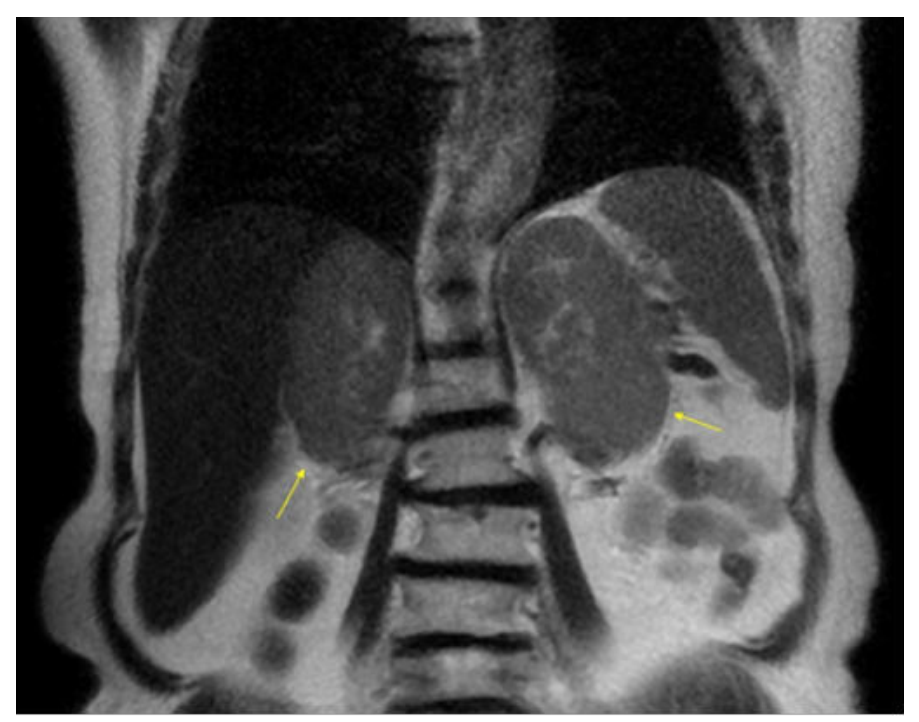

Fig. 2. Coronal T2-weighted images, hyperintense mass lesions of uniform character with smooth borders are observed in bilateral adrenal glands.

\section{DISCUSSION}

Adrenal masses are seen more frequently with the development of imaging methods.Bilateral adrenal masses constitute approximately $15 \%$ of the adrenal masses detected incidentally [3]. Granulomatous diseases, hemorrhage, metastatic lesions, or lymphoma should be suspected when bilateral, bulky masses are detected in the adrenal gland [4]. Bilateral primary adrenal lymphoma (PAL) of the adrenal gland is very rare. Its diagnosis is challenging, the prognosis is aggressive, and there is no specific laboratory test to make a diagnosis. LDH may be found to increase at a rate of $88 \%$, especially in patients with bilateral masses [2]. However, the LDH level was within the normal reference range in our patient. Just as there is no laboratory finding to diagnose PAL, there are no specific symptoms either. The patient can also present with nonspecific findings such as abdominal pain, weight loss, and adrenal insufficiency-related presentations such as hypotension, fever, fatigue, and hyperpigmentation. The adrenal cortical insufficiency occurs more often when $90 \%$ of the adrenal parenchyma is destructed in advanced primary disease stages [5]. Systemic symptoms such as fever, weight loss, and night sweating in malignancies are called Bsymptoms, and they are generally seen more in patients with large bilateral masses than the unilateral and small ones. This phenomenon has been attributed to tumor burden and cytokine storm released from lymphoid cells [2]. However, although our patient had massive bilateral masses, there were no B-symptoms contrary to this information.

Radiographic features are beneficial in the differential diagnosis of adrenal masses. The most commonly used imaging methods are Computed tomography (CT) and MRI. Through its ease of access, low cost, and short examination time, CT becomes prominent as the first test to evaluate adrenal lesions. In unenhanced $\mathrm{CT}$, the lesion being less than $10 \mathrm{HU}$, homogeneous, well-circumscribed, and small in diameter favors benign lesions, while as the size of the lesion increases, the likelihood of malignancy of the lesion increases as well. After excluding pheochromocytoma, $70 \%$ of the adrenal masses larger than 4 $\mathrm{cm}$ are malignant and this rate increases to $85 \%$ as the size increases over $6 \mathrm{~cm}$ [6]. Rapid loss of contrast (washout) in 10th-15th min post-contrast images in contrast-enhanced CT images is an essential indicator for detecting benign lesions. MRI and PET-CT are used in the differential diagnosis of adrenal lesions with low-fat content detected by CT. In adrenal lymphoma, there is no typical diagnostic image in imaging methods. In CT, nodular and diffuse thickening can be seen in the gland, imaging findings other than low density, low-moderate contrast overalls (no contrast washout and signal loss, diffusion restriction, and activity uptake) are that the hypointense pattern can be seen in T1 and hyperintense pattern in T2 in MRI; however, these findings are similar to other malignant diseases [7]. In our patient, there were no specific findings suggesting lymphoma on imaging. We predicted 
it was a malignant mass since the imaging findings of adrenal carcinoma include the large size of the lesion, heterogeneous and distinct contrast enhancement, $a b-$ sence of contrast washout phenomenon, no signal loss in chemical shift imaging, diffusion restriction in diffusion-weighted imaging, and activity uptake in PETCT images [8]. Based on the PET-CT findings, this patient was evaluated in favor of adrenal carcinoma and underwent adrenalectomy. Maybe a biopsy could be performed for this patient before the operation. However, the issue of biopsy in adrenal lesions is controversial. While some authors consider CT-guided biopsy as an easy and reliable method in cases with hesitation in diagnosis [8], some authors recommend biopsy only if it will change the course of oncological disease treatment with known malignancy. The sensitivity of adrenal biopsy performed with a pre-diagnosis of malignancy is $87 \%$, and the specificity is $100 \%$ [9].

A biopsy was not performed in our case; the patient underwent adrenalectomy based on imaging methods, considering adrenal carcinoma in the pre-diagnosis. The diagnosis of the disease was made by pathological examination. There were no distant metastases in the CT taken for staging after the operation. Based on these findings, our patient was diagnosed with PAL. PAL prognosis is generally poor, rapidly progressing, and aggressive. Although it initially appears to respond well to treatment, permanent remission is rare [10]. The average 1-year survival is less than $20 \%$, so it should be treated aggressively. There is no specific treatment regimen for PAL. Through CHOP and similar treatments with or without rituximab, survival is between $20-50 \%$. There is no difference in survival in the disease being unilateral and bilateral, as well as no difference in terms of treatment between undergoing surgery (adrenalectomy) or only chemotherapy. Information on the effectiveness of radiotherapy is very limited, and larger studies are needed.

\section{CONCLUSION}

As a result, lymphoma associated with the adrenal gland is extremely rare and difficult to diagnose. First of all, the clinician should have a high degree of suspicion in patients with adrenal insufficiency and bilat- eral adrenal mass. The diagnosis is made by imagingguided biopsy, surgical resection, or autopsy. Since its prognosis is poor, the diagnosis should be made quickly, and treatment should be started as soon as possible.

\section{Authors' Contribution}

Study Conception: SÇA; Study Design: SÇA; Supervision: SÇA; Funding: SÇA; Materials: SÇA; Data Collection and/or Processing: UA; Statistical Analysis and/or Data Interpretation: UA; Literature Review: SÇA; Manuscript Preparation: SÇA and Critical Review: SÇA.

\section{Conflict of interest}

The authors disclosed no conflict of interest during the preparation or publication of this manuscript.

\section{Informed consent}

Written informed consent was obtained from the patient for publication of this case and any accompanying images.

\section{REFERENCES}

1. Wang J, Sun NC, Renslo R, Chuang CC, Tabbarah HJ, Barajas $\mathrm{L}$, et al. Clinically silent primary adrenal lymphoma: a case report and review of the literature. Am J Hematol 1998;58:130-6.

2. Rashidi A, Fisher SI. Primary adrenal lymphoma: a systematic review. Ann Hematol 2013;92:1583-93.

3. Bourdeau I, El Ghorayeb N, Gagnon N, Lacroix A. Management of endocrine disease: differential diagnosis, investigation, andtherapy of bilateral adrenal incidentalomas. Eur J Endocrinol 2018;179:R57-R67.

4. Levy NT, Young WF, Habermann TM, Stricker JG, Carney JA, Stanson AW. Adrenal insufficiency as a manifestation of disseminated non-Hodgkin's lymphoma. Mayo Clin Proc 1997;72:81822.

5. Rosenthal FD, Davies MK, Burden AC. Malignant disease presenting as Addison's disease. Br Med J 1978;1:1591-2.

6. Turkey Endocrinology and Metabolism Diseases Society of Adrenal and Gonadal Guide 2019:p.67.

7. Anis M, Irshad A. Imaging of abdominal lymphoma. Radiol Clin North Am 2008;46:265-85.

8. Allolio B, Fassnacht M. Clinical review: an adrenocortical carcinoma-clinical update. J Clin Endocrinol Metab 2006;91:202737.

9. Fassnacht M, Arlt W, Bancos I, Dralle H, Newell-Price J, Sahdev A, et al. Management of adrenal incidentalomas: European Society of Endocrinology Clinical Practice Guideline in collaboration with the European Network for the Study of Adrenal 
Tumors. Eur J Endocrinol 2016;175: G1-G34.

10. Grigg AP, Connors JM. Primary adrenal lymphoma. Clin

Lymphoma 2003;4:154-60. 